\title{
On Evaluation of Video Quality Metrics: an HDR Dataset for Computer Graphics Applications
}

\author{
Martin Čadík, Tunç O. Aydın, Karol Myszkowski, Hans-Peter Seidel \\ MPI Informatik
}

\begin{abstract}
In this paper we propose a new dataset for evaluation of image/video quality metrics with emphasis on applications in computer graphics. The proposed dataset includes LDR-LDR, HDR-HDR, and HDR-LDR reference-test video pairs with various types of distortions. We also present an example evaluation of recent image and video quality metrics that were applied in the field of computer graphics. In this evaluation all video sequences were shown on an HDR display, and subjects were asked to mark the regions where they saw differences between test and reference videos. As a result, we capture not only the magnitude of distortions, but also their spatial distribution. This has two advantages: on one hand the local quality information is valuable for computer graphics applications, on the other hand the subjectively obtained distortion maps are easily comparable to the maps predicted by quality metrics.
\end{abstract}

\section{INTRODUCTION}

Experimental evaluation of computer graphics $(\mathrm{CG})$ techniques is necessary to validate their impact on perceived quality of resulting images. So far such image quality evaluation in CG is mostly performed informally without referring to well-established subjective and objective methods, which are commonly used in other fields, such as in image compression. In particular, CG field could benefit greatly from objective quality metrics due to the simplicity of their use and low costs involved. This however, requires extensive perceptual validation of such image and video quality metrics, which should be sensitive to image artifacts and distortions specific in CG. Another important aspect of such validation is high dynamic range (HDR) of images that are often generated by HDR rendering pipelines, which are today common in computer games (utilizing GPU or specialized consoles) and computer-aided design systems (in particular dealing with realistic image synthesis).

To make the validation (or calibration) of image or video quality metric possible, one needs to design a set of input stimuli (i.e. a dataset) and perform a user study which results in a set of subjective (mean) opinion scores. Subjective studies are very laborious and may be stimuli dependent, thus the community benefits from publicly available, standardized data sets. Therefore, a few datasets were published in the past. ${ }^{1-5}$

Unfortunately, none of the existing datasets is suitable for evaluation of video quality metrics in computer graphics field, where the images and videos often exhibit high dynamic range of luminance values and specific artifacts (see Figures 1,2). Existing datasets are limited in dynamic range of the input stimuli (only lowdynamic (LDR) range videos), in the distortions they cover (mostly compression-related artifacts), and in the extent of subjective responses (usually the numerical rating of the quality of the stimulus). Few authors employed a concept of image distortion maps ${ }^{6-8}$ in evaluation of image quality metrics, but this has not been done for temporal distortions in videos so far. To overcome the above limitations, we propose and make publicly available* a new dataset for evaluation of image/video quality metrics with emphasis on applications in computer graphics. Several aspects were influential while designing the dataset: (i) in addition to the assessmet of the quality of LDR videos, the assessment of high-dynamic range videos, as well as comparing HDR videos with LDR videos and vice versa, and (ii) the outcome of the subjective experiment in the form of distortion maps that show quality prediction as a function of spatial position which is especially important for applications in computer graphics. Furthermore, we show an example evaluation of recent image and video quality metrics that were

Further author information: e-mail: \{mcadik, tunc, karol, hpseidel\}@mpi-inf.mpg.de Max Planck Institut Informatik, Saarbrücken

*http://www.mpi-inf.mpg.de/resources/hdr/quality 
applied in the field of computer graphics. The goal of this evaluation was to examine the correlation between the objective quality predictions computed by the video quality metrics, and the subjective responses obtained by the experimental procedure. It is known ${ }^{9}$ that applications of image/video quality metrics into the field of computer graphics are still far from maturity, we believe however, that the published dataset helps in validation and improvement of existing, and the design of future metrics for computer graphics and other applications.

To that end the proposed dataset and the subjective study have the following unique features over previous studies on video quality assessment:

- The test set includes LDR-LDR, HDR-HDR, and HDR-LDR reference-test video pairs with various types of distortions.

- A BrightSide DR37-P HDR display (max. luminance $\approx 3000 \mathrm{~cd} / \mathrm{m}^{2}$ ) was used for displaying the videos.

- The subjects were not asked to assess only an overall quality of the video, but to mark the regions where they saw differences between test and reference videos, resulting in distortion maps similar to the metric outcome.

In the remainder of this paper we describe the proposed dataset for evaluation of video quality metrics, the experimental setup and procedure (Section 2), present an example evaluation of video quality metrics using the dataset (Section 3) and discuss the results based on the correlation between the outcome of the subjective data and corresponding predictions of state-of-the art video quality metrics.
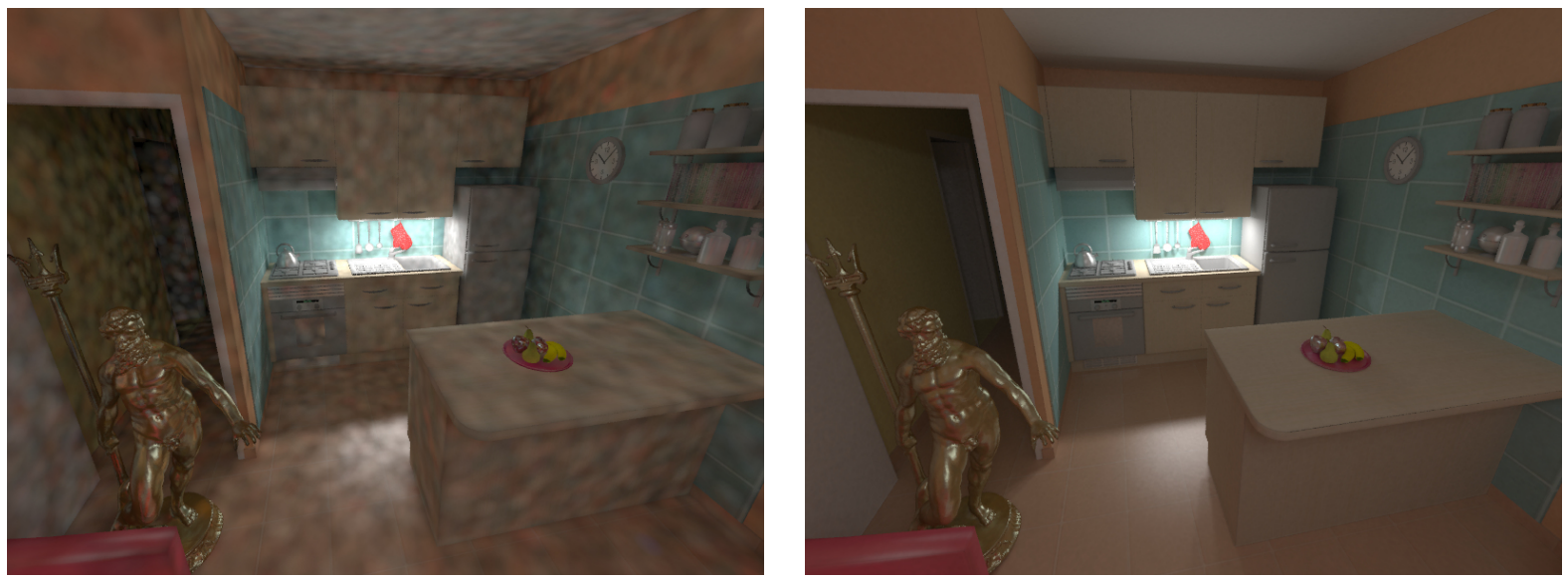

Figure 1. An example of typical artifacts in rendered images and video sequences: an indoor scene rendered using progressive photon mapping algorithm. ${ }^{10}$ Left: non-converged solution (2 iterations) exhibits low-frequency noise. Right: fully converged solution.

\section{DATASET FOR VIDEO METRIC EVALUATION}

The proposed dataset consists of 9 reference-test video pairs (1 LDR-LDR, 2 HDR-LDR, and 6 HDR-HDR), they are listed in Table 1 . The video stimuli were generated by imposing temporally varying visual artifacts to HDR scenes (Figure 3), such as HDR video compression artifacts and temporal random noise along with temporal luminance modulation and tone mapping. The magnitudes of the visual artifacts were carefully selected so that there were sub-, near- and supra-threshold distortions present in the experimental videos. In sequences \#1$\# 4$, and \#9 the temporal random noise was generated by filtering a three dimensional array of random values between -0.5 and 0.5 by a Gaussian with standard deviations 20 (referred as "high stddev") and 5 (referred as "low stddev") pixels along each dimension. The magnitude of noise was adjusted by multiplying with two constants separately, such that the artifacts are barely visible in one setting (referred as "low magnitude"), and clearly visible in the other (referred as "high magnitude"). In sequences \#5 and \#6, the HDR compression ${ }^{13}$ 

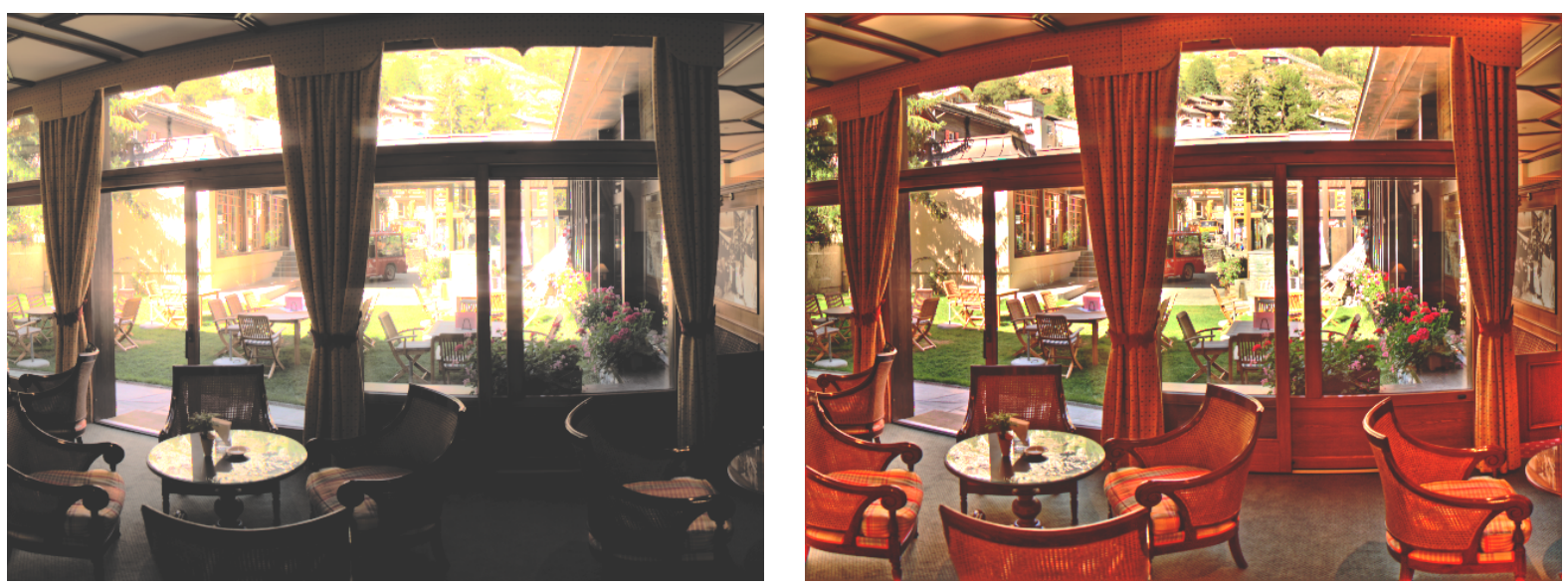

Figure 2. An example of artifacts due to the tone mapping of HDR images and HDR video sequences. Left: global tone mapping technique by Pattanaik et al. ${ }^{11}$ preserves overall image contrast, but results in severe loss of details. Right: gradient-based technique of Fattal et al. ${ }^{12}$ is able to reproduce virtually all the image details, at the cost of an overall contrast and contrast reversals (i.e. halo artifacts).

was similarly applied at two levels to the HDR scenes, where the luminance was globally modulated over time by $0.5 \%$ of the maximum scene luminance to vary the visibility of image details over time. Videos generated by applying tone mapping operators ${ }^{11,12}$ to each input HDR video frame were used in the dynamic range independent comparisons (sequences \#7 and \#8).

All test videos consist of 60 frames, and should be presented at 24 fps. In order to faithfully reproduce the luminance values on the HDR display, the response function of the display was measured using a Minolta LS100 luminance meter. The measurements consisted of 17 samples taken from the displayable luminance range. The sample points were then fitted to a $3^{\text {rd }}$ degree polynomial function, from which 100 points were resampled and stored as a lookup table. Finally, the pixel values for the HDR videos were determined by cubic spline interpolation between nearest two luminance levels. Furthermore, the displayed luminance of the HDR videos were measured again at various regions, and whenever necessary, the scenes were slightly recalibrated to ensure that the displayed luminance values match the actual scene luminance.

\begin{tabular}{|c|c|c|c|c|}
\hline$\#$ & Source & Ref. DR & Test DR & Artifact Type of Test Video \\
\hline 1 & Cars & HDR & HDR & Noise - high magnitude, low stddev \\
\hline 2 & Lamp & HDR & HDR & Noise - high magnitude, low stddev \\
\hline 3 & Desk & HDR & HDR & Noise - low magnitude, low stddev \\
\hline 4 & Tree & HDR & HDR & Noise - high magnitude, high stddev \\
\hline 5 & Cafe & HDR & HDR & HDR compression - high quality, luminance mod. \\
\hline 6 & Tower & HDR & HDR & HDR compression - low quality, luminance mod. \\
\hline 7 & Cafe & HDR & LDR & Luminance modulation, Pattanaik's tone mapping \\
\hline 8 & Lamp & HDR & LDR & Luminance modulation, Fattal's tone mapping \\
\hline 9 & Lamp & LDR & LDR & Noise - low magnitude, low stddev \\
\hline
\end{tabular}

Table 1. List of the experimental stimuli. Refer to text for details.

The participants of the experimental study were 16 subjects of age 23 to 50 . They all had near-perfect or corrected to normal vision, and were naïve for the purposes of the experiment. Each subject evaluated the quality of the whole test set through a graphical user interface displayed on a BrightSide DR37-P HDR display (Figure 4). In the HDR-HDR, and LDR-LDR comparisons, the task was to mark the regions in the test video where visible differences were present with respect to the reference video. In the HDR-LDR comparisons on the other hand, the subjects were asked to assess the contrast loss and amplification. In the instruction phase 


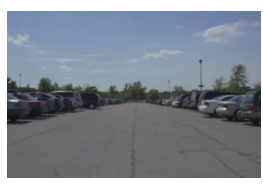

Cars

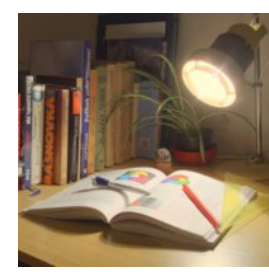

Lamp

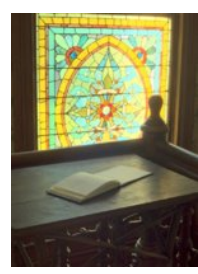

Desk

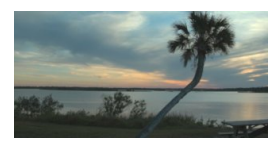

Tree

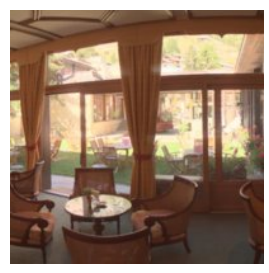

Cafe

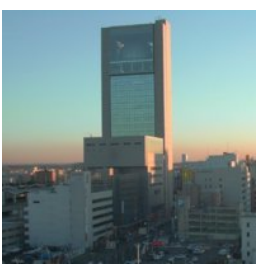

Tower

Figure 3. The video test set is generated from 6 calibrated HDR scenes (tone mapped for presentation purpose ${ }^{14}$ ). The scene luminance was clipped where it exceeded the maximum display luminance. The displayed luminance of the videos resulting from the scenes were between 0.1 and $3000 \mathrm{~cd} / \mathrm{m}^{2}$.

before the experiment, the subjects were asked to mark a grid tile even if visible differences were present only in a portion of that grid's area. They were also encouraged to mark a grid tile in the case they cannot decide whether it contains a visible difference or not. The subjects were placed 0.75 meters away from the display so that a $512 \times 512$ image spanned 16 visual degrees and the grid cell size was approximately 1 visual degree. The environment illumination was dimmed and controlled, and all subjects were given time to adapt to the room illumination. There were no time limitations set for the experiment, but the majority of the subjects took 15-30 minutes for the entire test set.

The marked regions for each trial were stored as distortion maps with $16 \times 16$ resolution, which were then averaged over all subjects to find the mean subjective response, see Figure 6 (first column). The descriptive statistics of these maps are summarized in Table 2 (first column). Figure 5 shows the standard deviation for each stimulus over the test subjects, separately for each grid tile. Over all images, the minimum and maximum values are obtained as 0 and 0.51 , the former indicating the tiles on which all subjects gave the same response, and the latter indicating the tiles where approximately half of the subjects have marked.
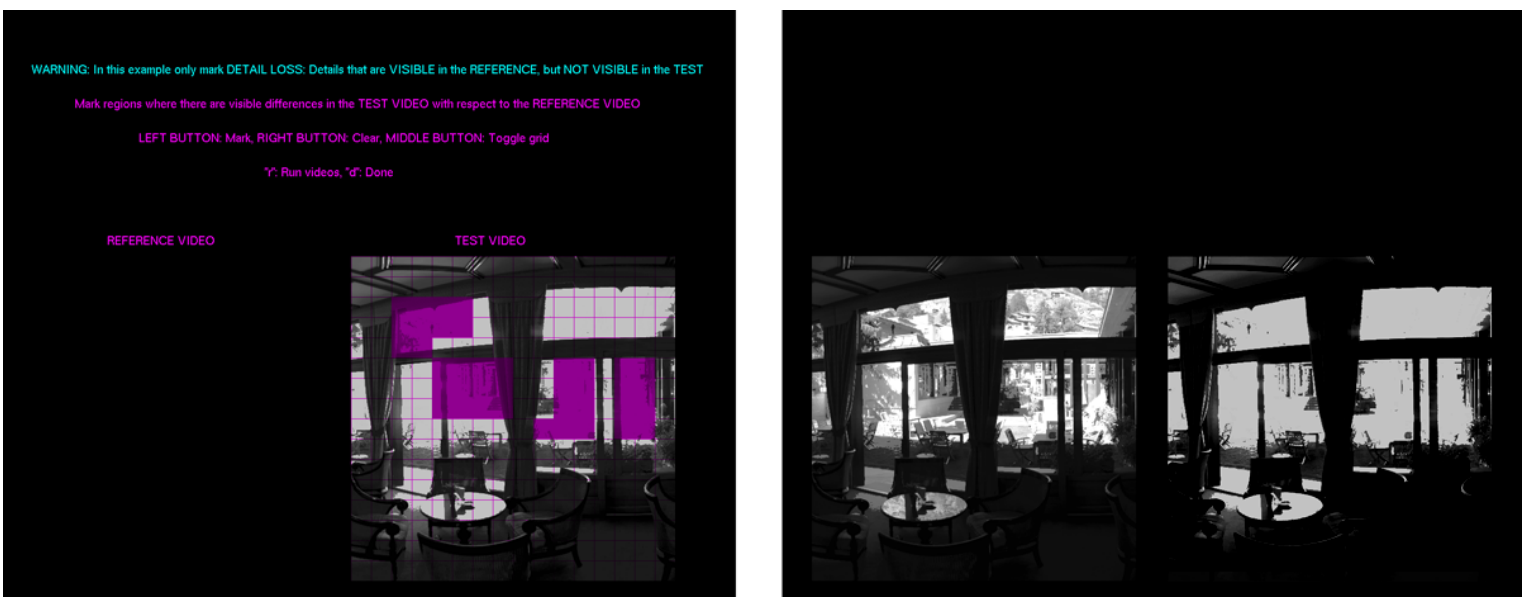

Figure 4. The experiment was performed through a graphical user interface on the HDR display. Subjects were shown reference and test videos side by side in a randomized order (right), and were asked to mark the relevant image locations on a $16 \times 16$ grid according to the instructions (left). The interface and messages were disabled while the videos were being shown. The interface allowed the subjects to watch the videos for an unlimited amount of iterations.

\section{EXAMPLE EVALUATION}

To illustrate the utilization of the proposed dataset, we show an evaluation of four state-of-the-art image/video quality metrics: DRIVQM, ${ }^{15}$ PDM,${ }^{16}$ HDRVDP,${ }^{8}$ and DRIVDP.${ }^{17}$ For each of the evaluated metrics the predictions for each stimulus were calculated, averaged over the whole 60 frames, and downsampled to the same 


\begin{tabular}{|l|l|l|l|l|l|}
\hline Stimulus \# & Subjective Response & DRIVQM & PDM & HDRVDP \\
\hline & {$[\mathrm{min}, \mathrm{max}] ;$ avg; std } & {$[\mathrm{min}, \mathrm{max}] ;$ avg; std } & {$[\mathrm{min}, \mathrm{max}] ;$ avg; std } & {$[\mathrm{min}, \mathrm{max}] ;$ avg; std } & {$[\mathrm{min}, \mathrm{max}] ;$ avg; std } \\
\hline 1 & {$[0.000,1.000] ; 0.177 ; 0.276$} & {$[0.000,0.850] ; 0.128 ; 0.230$} & {$[0.000,0.301] ; 0.082 ; 0.079$} & {$[0.000,0.019] ; 0.001 ; 0.002$} & {$[0.075,0.417] ; 0.194 ; 0.058$} \\
\hline 2 & {$[0.000,1.000] ; 0.201 ; 0.347$} & {$[0.000,0.954] ; 0.185 ; 0.282$} & {$[0.000,0.813] ; 0.061 ; 0.138$} & {$[0.000,0.893] ; 0.050 ; 0.157$} & {$[0.072,0.799] ; 0.218 ; 0.155$} \\
\hline 3 & {$[0.000,1.000] ; 0.082 ; 0.242$} & {$[0.000,0.307] ; 0.015 ; 0.045$} & {$[0.000,0.052] ; 0.003 ; 0.008$} & {$[0.000,0.889] ; 0.163 ; 0.247$} & {$[0.006,0.440] ; 0.090 ; 0.078$} \\
\hline 4 & {$[0.000,1.000] ; 0.124 ; 0.250$} & {$[0.001,0.457] ; 0.094 ; 0.115$} & {$[0.000,0.024] ; 0.007 ; 0.006$} & {$[0.000,0.000] ; 0.000 ; 0.000$} & {$[0.067,0.240] ; 0.137 ; 0.039$} \\
\hline 5 & {$[0.000,1.000] ; 0.066 ; 0.186$} & {$[0.000,0.420] ; 0.026 ; 0.063$} & {$[0.000,0.952] ; 0.146 ; 0.207$} & {$[0.000,0.866] ; 0.074 ; 0.166$} & {$[0.040,0.873] ; 0.241 ; 0.199$} \\
\hline 6 & {$[0.000,1.000] ; 0.399 ; 0.389$} & {$[0.072,0.468] ; 0.232 ; 0.103$} & {$[0.810,0.984] ; 0.965 ; 0.026$} & {$[0.180,0.942] ; 0.657 ; 0.202$} & {$[0.626,0.928] ; 0.789 ; 0.058$} \\
\hline 7 & {$[0.000,1.000] ; 0.312 ; 0.392$} & {$[0.037,0.984] ; 0.451 ; 0.342$} & {$[0.838,0.984] ; 0.980 ; 0.018$} & {$[0.002,0.953] ; 0.448 ; 0.327$} & {$[0.031,0.953] ; 0.374 ; 0.288$} \\
\hline 8 & {$[0.000,0.812] ; 0.108 ; 0.180$} & {$[0.041,0.942] ; 0.225 ; 0.146$} & {$[0.606,0.984] ; 0.971 ; 0.043$} & {$[0.005,0.953] ; 0.509 ; 0.274$} & {$[0.148,0.884] ; 0.406 ; 0.172$} \\
\hline 9 & {$[0.000,1.000] ; 0.105 ; 0.238$} & {$[0.000,0.502] ; 0.054 ; 0.104$} & {$[0.000,0.396] ; 0.032 ; 0.066$} & {$[0.000,0.211] ; 0.006 ; 0.025$} & {$[0.067,0.577] ; 0.176 ; 0.097$} \\
\hline
\end{tabular}

Table 2. Descriptive statistics of distortion maps (depicted in Figure 6) for each input stimulus. Abbreaviations used: $\min =$ minimal value, $\max =$ maximal value, avg=average value, std=standard deviation, of the distortion map averaged over all subjects/metric responses for a particular stimulus (1-9).

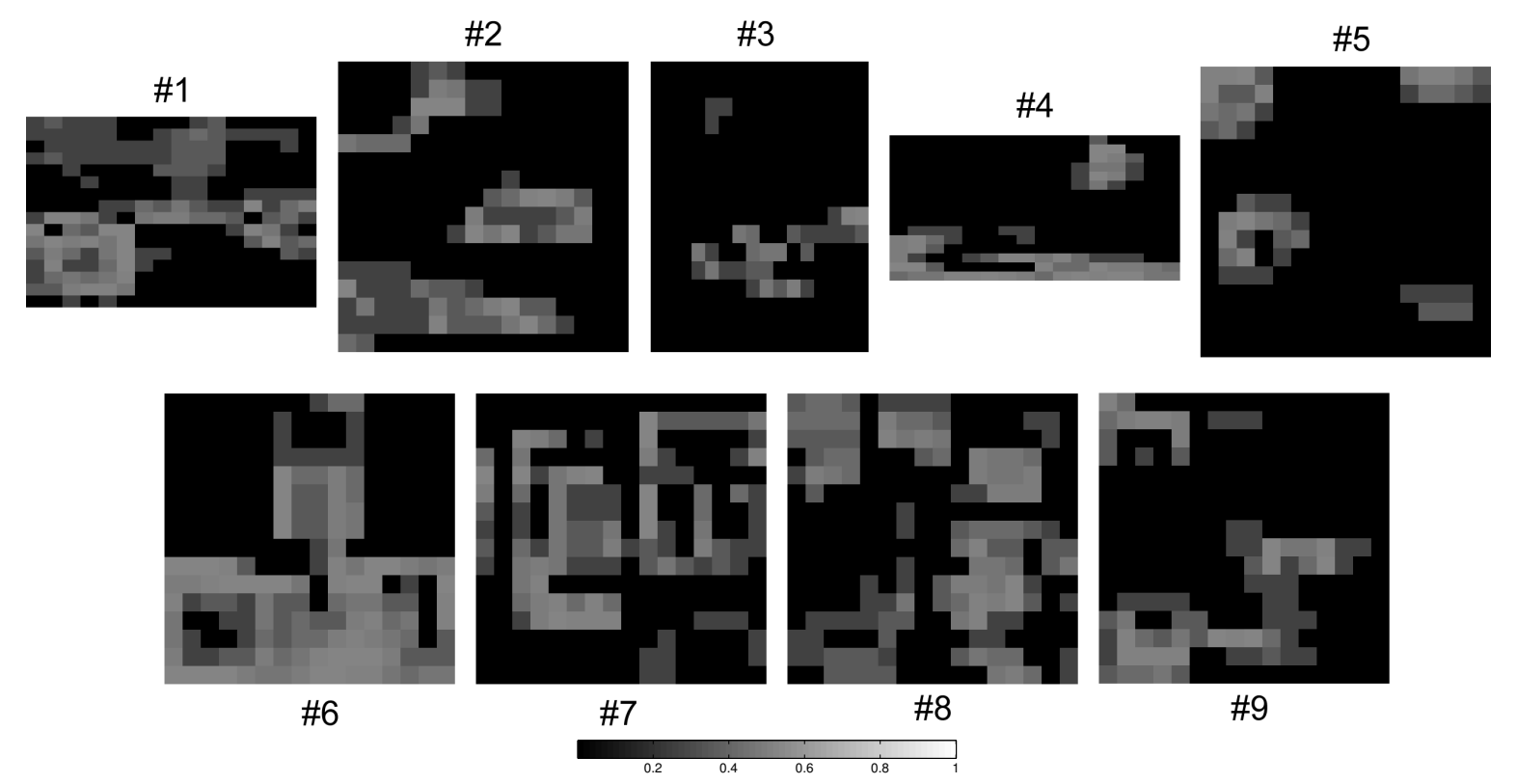

Figure 5. Maps showing the standard deviations over subjects for each stimulus. The numbers refer to the first column of Table 1.

resolution as the mean subjective response. In all the tests in this paper we used a frequency domain implementation of DRIVQM metric, precisely following the reference publication. We found that using this implementation was still feasible for the frame sizes and durations of the video sequences in our dataset. For larger sequences we also implemented an alternative version of the metric, where the frequency domain channel decomposition is replaced by a Steerable Pyramid based spatial decomposition (described in Appendix A). A web service based on this implementation is publicly available at http://drivqm.mpi-inf.mpg.de. The HDRVDP and DRIVDP metrics are designed for image quality evaluation, thus the video stimuli were evaluated for each frame separately. For each video pair, we computed the $2 \mathrm{D}$ correlation between the mean subjective response and the metric prediction (Table 3) and used the results to evaluate the performance of the metrics. The Figure 6 shows the mean subjective distortion maps along with the corresponding metrics predictions for visual inspection. The descriptive statistics of these maps are summarized in Table 2.

For the purposes of generating the maps in Figure 6, in cases of PDM and HDRVDP we simply used the distortion maps produced by those metrics. In the DRIVDP case however, the output of the metric is three separate maps for contrast loss, amplification and reversal. Thus, it is not clear how to produce a single distortion map for HDR-HDR and LDR-LDR stimuli. After experimenting with various methods for combining 
the distortion maps predicted by DRIVDP, we found that the combined map defined as:

$$
P_{\text {combined }}^{k, l, m}=1-\left(1-P_{l o s s}^{k, l, m}\right) \cdot\left(1-P_{a m p l}^{k, l, m}\right),
$$

gives the best correlation with subjective data. Here, $P_{l o s s \mid a m p l}^{k, l, m}$ refer to the detection probability of contrast loss and amplification at scale $k$, orientation $l$, and temporal channel $m$. The resulting map $P_{c o m b i n e d}^{k, l, m}$ corresponds to the probability of detecting either contrast loss or amplification at a visual channel. Leaving contrast reversal resulted in slightly improved correlations.

\subsection{Discussion}

As DRIVQM is the only evaluated metric that was designed specifically for the purposes of dynamic rangeindependent video quality assessment of computer graphics sequences, it is not surprising that it overcomes the other metrics in most cases. Highest correlations were obtained for the \#2 HDR-HDR Lamp stimulus with high magnitude, low standard deviation noise, and the \#7 HDR-LDR Cafe stimulus with luminance modulation and Pattanaik's tone mapping (0.883 and 0.879 , respectively). For these two cases, the magnitude of the probability of detection predicted by the metric, and the average of the binary maps over subjects obtained experimentally are also very similar. In other cases, either the magnitudes of the mean subjective maps were lower than the corresponding detection probability magnitude predictions (such as \#4 Tree HDR-HDR stimulus with high magnitude, high standard deviation noise, and \#9 Lamp LDR-LDR stimulus with noise), or a certain region with visible distortions was missed out (\#1 Cars HDR-HDR stimulus with high magnitude, low standard deviation noise). For the remaining stimuli, a combination of both deviations can be observed in the metric predictions and subjective responses. The worst prediction of DRIVQM is $(\# 8,0.733)$.

HDRVDP, while capable of evaluating the quality of HDR images, lacks any temporal processing and is geared towards comparing images with the same dynamic range. The DRIVDP addresses the latter limitation, but still suffers from the former. Consequently, DRIVDP's predictions for the HDR-LDR stimuli (numbers 7 and 8 ) is slightly better than HDRVDP. PDM, on the other hand, is designed for the video stimuli, but lacks the HDR and dynamic range independent mechanisms of HDRVDP and DRIVDP, producing the least average correlation with the subjective responses. As shown in Table 3, DRIVQM significantly outperforms others in most cases. The significant difference in average correlations over the entire test set (last row of Table 3) shows that overall DRIVQMs predictions are clearly more accurate than others. The corresponding distortion maps predicted by PDM, HDRVDP and DRIVDP are shown in Figure 6 columns $3-5$ (averaged and downsampled to $16 \times 16$ after the computation).

While the relation between the correlation values and distortion maps is obvious in most cases, the high correlation of PDM for stimulus \#3 deserves further explanation. While PDM correctly detects the distorted regions in that stimulus in a spatial sense, the magnitude of detection probabilities are very low (refer to Table 2), to the point that they are quantized by the visualization. Thus the map appears to be blank, but since the relation with the subjective data is linear, the correlation is high.

\section{CONCLUSION}

The main goal of this work was to develop a dataset of video sequences accompanied by the corresponding subjective data evaluating their quality in a local manner. Such locality is the key in computer graphics applications, where local image distortions should be detected and if possible corrected in rendering. Another important aspect of such dataset is dynamic range of frames, where pairs of HDR, LDR and mixed LDR-HDR reference and test videos, which are calibrated in terms of pixel luminance, are considered. We propose also a novel subjective testing setup that involves an HDR display, which is suitable for reproducing luminance levels in the videos, as well as interactive marking of local image regions where distortions are visible.

The dataset proved to be useful in calibrating and validating the DRIVQM, ${ }^{15}$ which has been developed specifically for computer graphics applications. The dataset is publicly available (http://www.mpi-inf.mpg. $\mathrm{de} / \mathrm{resources} / \mathrm{hdr} /$ quality) and our hope is that it can be used in other validation tasks. 


\begin{tabular}{|c|c|c|c|c|}
\hline Stimulus \# & DRIVQM & PDM & HDRVDP & DRIVDP \\
\hline 1 & $\mathbf{0 . 7 6 5}$ & -0.0147 & 0.591 & 0.488 \\
\hline 2 & $\mathbf{0 . 8 8 3}$ & 0.686 & 0.673 & 0.859 \\
\hline 3 & 0.843 & $\mathbf{0 . 8 8 6}$ & 0.0769 & 0.865 \\
\hline 4 & $\mathbf{0 . 8 1 5}$ & 0.0205 & 0.211 & -0.0654 \\
\hline 5 & $\mathbf{0 . 8 4 4}$ & 0.565 & 0.803 & 0.689 \\
\hline 6 & $\mathbf{0 . 7 6 1}$ & -0.462 & 0.709 & 0.299 \\
\hline 7 & 0.879 & 0.155 & 0.882 & $\mathbf{0 . 9 2 4}$ \\
\hline 8 & $\mathbf{0 . 7 3 3}$ & 0.109 & 0.339 & 0.393 \\
\hline 9 & $\mathbf{0 . 7 5 3}$ & 0.368 & 0.473 & 0.617 \\
\hline Average & $\mathbf{0 . 8 0 9}$ & 0.257 & 0.528 & 0.563 \\
\hline
\end{tabular}

Table 3. Correlations of subjective responses with predictions of DRIVQM, PDM, HDRVDP, and DRIVDP. The last row shows the average correlations over the test set, the best correlations for each stimulus are printed in bold text.

\section{APPENDIX A. EFFICIENT IMPLEMENATATION OF DRIVQM}

As we admit in the discussion section of the previous publication, ${ }^{15}$ executing DRIVQM becomes infeasible for long video sequences due to the long processing time. Moreover, the 64-frames window size practically means that for frame sizes larger than VGA the memory consumption is prohibitively large for an average desktop computer. As suggested by the authors of the metric, we replace the frequency domain Cortex Transform by the Steerable Pyramid ${ }^{18}$ with 6 levels (where each differ by one octave) and 6 orientations, and use Winkler's ${ }^{19}$ 9-tap approximation of the transient and sustained temporal mechanisms. The base band of the Steerable Pyramid, analogous to Cortex Transform, does not have any orientations. For the purpose of accounting for spatial phase uncertainty, the Hilbert transforms of the steerable filters provided by Freeman and Adelson's formulation are used, whereas temporal phase uncertainty is ignored. All other components of the DRIVQM are left intact, including the extended Contrast Sensitivity Function. The updated implementation is more efficient in memory consumption and running time, without any significant deviation in results from the original implementation. A publicly available web service that uses our updated implementation can be found at http: //drivqm.mpi-inf.mpg.de. The web service requires the users to upload the frames of their test and reference video pair of either the same dynamic range, or different dynamic ranges. Users are allowed to change metric parameters, such as the pixels per visual degree, frames per second and adaptation luminance. Once the setup is complete, the metric is run on the uploaded video pair. Upon completion, the input video pair is immediately deleted, and the user is provided with a link to the contrast difference, contrast loss, and contrast amplification maps.

\section{ACKNOWLEDGMENTS}

The authors would like to thank all the staff members and students at MPI Informatik who kindly participated in the subjective experiments.

\section{REFERENCES}

[1] Carney, T., Klein, S. A., Tyler, C. W., Silverstein, A. D., Beutter, B., Levi, D., Watson, A. B., Reeves, A. J., Norcia, A. M., Chen, C.-C., Makous, W., and Eckstein, M. P., "The development of an image/threshold database for designing and testing human vision models," in [Proc. of Human Vision, Visual Processing, and Digital Display IX], SPIE, Bellingham, WA, 3644 (1999).

[2] The Video Quality Experts Group, "Final report from the video quality experts group on the validation of objective quality metrics for video quality assessment, Phase I." http://www.its.bldrdoc.gov/vqeg/ projects/frtv (2000).

[3] Sheikh, H. R. and Bovik, A. C., "LIVE image quality assessment database." http://live.ece.utexas. edu/research/quality/subjective.htm (2003). 
[4] Seshadrinathan, K., Soundararajan, R., Bovik, A. C., and Cormack, L. K., "LIVE video quality database." http://live.ece.utexas.edu/research/quality/live_video.html (November 2009).

[5] Seshadrinathan, K., Soundararajan, R., Bovik, A. C., and Cormack, L. K., "A subjective study to evaluate video quality assessment algorithms," Human Vision and Electronic Imaging XV 7527(1), 75270H, SPIE (2010).

[6] Zhang, X., Setiawan, E., and Wandell, B., "Image distortion maps," in [Fifth Color Imaging Conference: Color Science, Systems and Applications], 120-125 (1997).

[7] Zhang, X. and Wandell, B. A., "Color image fidelity metrics evaluated using image distortion maps," Signal Processing 70(3), $201-214$ (1998).

[8] Mantiuk, R., Daly, S., Myszkowski, K., and Seidel, H.-P., "Predicting visible differences in high dynamic range images - model and its calibration," in [Human Vision and Electronic Imaging X], SPIE Proceedings Series 5666, 204-214 (2005).

[9] Rogowitz, B. E. and Rushmeier, H. E., "Are image quality metrics adequate to evaluate the quality of geometric objects?," in [Proc. of Human Vision and Electronic Imaging VI], 340-348, SPIE (2001).

[10] Hachisuka, T., Ogaki, S., and Jensen, H. W., "Progressive photon mapping," in [ACM Transactions on Graphics (Proc. of SIGGRAPH Asia'08)], 27(5), 1-8, ACM, New York, NY, USA (2008).

[11] Pattanaik, S. N., Tumblin, J. E., Yee, H., and Greenberg, D. P., "Time-dependent visual adaptation for fast realistic image display," in [ACM Transactions on Graphics (Proc. of ACM SIGGRAPH'00)], 47-54, ACM Press (2000).

[12] Fattal, R., Lischinski, D., and Werman, M., "Gradient domain high dynamic range compression," in $[A C M$ Transactions on Graphics (Proc. of ACM SIGGRAPH'02)], 249-256, ACM Press (2002).

[13] Mantiuk, R., Krawczyk, G., Myszkowski, K., and Seidel, H.-P., "Perception-motivated high dynamic range video encoding," in [ACM Transactions on Graphics (Proc. of SIGGRAPH'04)], 23(3), 733-741, ACM (2004).

[14] Reinhard, E., Stark, M., Shirley, P., and Ferwerda, J., "Photographic tone reproduction for digital images," in [ACM Transactions on Graphics (Proc. of SIGGRAPH'02)], 267-276, ACM Press (2002).

[15] Aydın, T. O., Čadík, M., Myszkowski, K., and Seidel, H.-P., "Video quality assessment for computer graphics applications," in [ACM Transactions on Graphics (Proc. of SIGGRAPH Asia'10)], 1-10, ACM, Seoul, Korea (2010).

[16] Winkler, S., [Digital Video Quality: Vision Models and Metrics], Wiley (2005).

[17] Aydın, T. O., Mantiuk, R., Myszkowski, K., and Seidel, H.-P., "Dynamic range independent image quality assessment," in [ACM Transactions on Graphics (Proc. of ACM SIGGRAPH'08)], 27(3) (2008). Article 69.

[18] Freeman, W. T. and Adelson, E. H., "The design and use of steerable filters," Pattern Analysis and Machine Intelligence, IEEE Transactions on 13(9), 891-906 (1991).

[19] Winkler, S., "A perceptual distortion metric for digital color video," in [Proc. of Human Vision and Electronic Imaging, SPIE], Controlling Chaos and Bifurcations in Engineering Systems 3644, 175-184, IEEE (1999). 


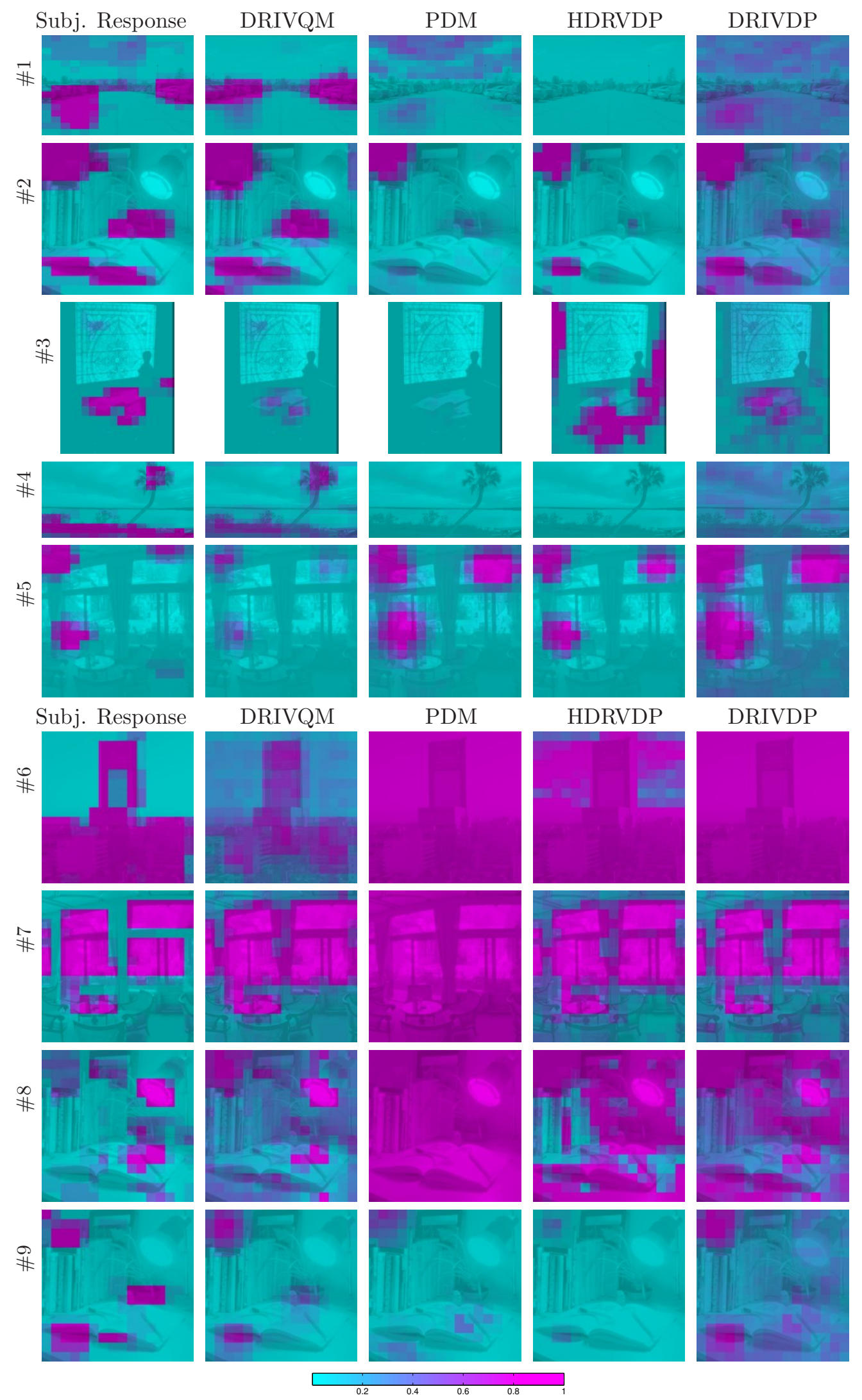

Figure 6. Mean subjective response distortion maps and corresponding mean metric predictions pairs. 